\title{
Method of Solving Multi-Index Distribution Tasks with Fuzzy Parameters*
}

\author{
Alexander Bozhenyuk \\ Academy for Engineering and Technologies \\ Southern Federal Universitý (SFEDU) \\ Taganrog, Russia \\ e-mail: avb002@yandex.ru
}

\author{
Olesya Kosenko \\ Academy for Engineering and Technologies \\ Southern Federal University (SFEDU) \\ Taganrog, Russia \\ e-mail: o_kosenko@mail.ru
}

\begin{abstract}
Resource allocation systems are characterized by a large number of elements and connections between them, a high degree of dynamism, which leads to the fact that the system operates under conditions of uncertainty of the external and internal environment. The need to solve high-dimensional distribution tasks in conditions of uncertainty and fuzziness requires the development of appropriate information and mathematical support. The method of solving multi-index transport problems taking into account the indeterminacy of the demand parameters and the cost of cargo transportation is considered in this article. A software application was developed to solve a multi-index distribution problem with fuzzy parameters. The software product is developed in the programming environment $1 \mathrm{C}$ v8.3. The article contains interface windows that implement the user's dialogue with the software application.
\end{abstract}

Keywords- multi-index distribution tasks, fuzzy optimization, approximate methods, uncertainty of source data, fuzzy interval method

\section{INTRODUCTION}

Distribution of resources is an important part of the production activities of enterprises. When managing the work of enterprises, workshops, equipment, methods are used to solve various kinds of distribution tasks. A common feature of these tasks is the allocation of resources between their producers and consumers of these resources in such a way that a group of pre-selected criteria is met [1]. Mechanisms for scheduling distribution processes are of particular importance in managing multi-stage technological processes of processing a resource that comes from the producer to the warehouse and then to the consumer.

Optimizing allocation of resources will be available within a company, but the maximum effect can be obtained by optimizing the total material flow throughout the primary resource from the source (manufacturer) to the end user [2]. At the same time, all links of the given resource allocation chain should work as a coherent mechanism. To solve such a problem, a systematic approach to the management of resource allocation, inventory management, demand forecasting, operational planning and management, as well as to logistics coordination is needed. Increasing the controlled distribution parameters of the problem allows taking into account more factors affecting the cost of moving resources

*This work has been supported by the Ministry of Education and Science of the Russian Federation under Project "Methods and means of decision making on base of dynamic geographic information models" (Project part, State task 2.918.2017)
[3].

Difficulties in the practical application of these methods to multiparametric resource allocation problems in the analysis of classical methods for solving distributive problems were identified. The uncertainty of the source data is not taken into account. Therefore, due to the large dimensionality of the problems of this class, it is difficult to obtain an exact solution for a permissible time. This circumstance determines the relevance of the development of simple and effective approximate methods for solving multi-index resource allocation problems that make it possible to obtain the best value for a permissible time in solving a large-dimensional problem and taking into account the uncertainty of the input data.

\section{DESCRIPTION OF METHOD}

The creation of a new distribution system that is stable with respect to external disturbances leads to the need to solve a number of specific problems, among which the following can be distinguished [4]:

- Estimation and forecasting of the demand for resources;

- Planning and organization of production of resources;

- Calculation of optimal levels of orders for resources and associated definition of volumes and organization of rational functioning of the transport subsystem;

\section{- Accounting for the uncertainty of the input data.}

The uncertainty of the initial parameters of the distribution problem is determined by fluctuations in demand, changes in resource transfer rates, distribution conditions, and inaccurate data. This circumstance predetermines the use of intelligent technologies. In the works $[5,6]$ proposed to account for uncertainty multiparametric initial data distribution tasks through the use of probabilistic models. However, due to the lack of information for the application of probabilistic models and the difficulties of operating random variables, it is proposed in [7] to use the fuzzy interval method, which allows effectively describing uncertainties in the form of intervals, and represents a convenient and adequate mathematical apparatus for studying such systems. Here, the parameters of the problem are given in the form of intervals at the boundaries of which certain parameters correspond to the parameters [8]. These boundaries are estimated with a 
sufficient degree of reliability according to statistical data, if they are accumulated enough to determine the parameters, or are set by experts on the basis of accumulated experience and intuitive assumptions. If we accurately determine the boundaries of interval estimates, then there is a possibility that the boundaries can be either overvalued or underreported, which will cause doubt in the results of calculations. Setting parameters in the form of a fuzzy interval will be both overestimated and underestimated, and the carrier of the fuzzy interval will be chosen so that the core of the fuzzy interval contains the most plausible values, and it will be guaranteed to find the parameter in question within the required limits.

A fuzzy interval is specified on the set $Q$ of the four parameters [9]:

$$
\widetilde{Q}=(\alpha, \beta, A, B)
$$

Here $\alpha$ is the left coefficient of fuzziness; $\beta$ is the right fuzzy coefficient; $A$ is the lower modal value; $B$ is the upper modal value of the fuzzy interval. Parameters $A$ and $B$ define the core of the fuzzy interval in the expression (1).

Based on the studies carried out, it was revealed that the classical model of the distribution problem does not fully describe the specifics of modern cargo transportation. The heterogeneity of resources and the variety of types of vehicles (transmission elements) are not always used in the description of the distribution system. Also, the possibility of allocating storage and distribution centers to reduce freight costs [10] is not considered, and the uncertainty and economic state of the environment are not taken into account. These parameters can be taken into account by considering the multi-index resource allocation tasks. To take them into account, variables with more than two indexes are entered.

Let's consider the five-index resource allocation problem, which allows to take into account the basic elements of the distribution system that determine the total cost of transportation of resources:

- Sources of resources;

- Consumers of resources;

- heterogeneous resources;

- transmitting elements of various types;

- centers of distribution and storage of resources.

The interconnection and interdependence of all elements of the distribution system necessitated an integrated approach to their study. To determine the parameters of the five-index resource allocation problem in the form of fuzzy intervals, we will present the function that determines the value of the resource allocation cost as a fuzzy function:

$$
\tilde{F}(X)=\sum_{i=1}^{I} \sum_{j=1}^{J} \sum_{k=1}^{K} \sum_{l=1}^{L} \sum_{r=1}^{R} \tilde{n}_{i j k l r} \tilde{x}_{i j k l r} \rightarrow \min ,
$$

where $\widetilde{\mathbf{C}}_{\mathrm{ijklr}}$ is a fuzzy set that is specified on the set of real numbers $\Theta_{c}$ and determines the fuzzy cost of transporting the unit of the $r$-th resource from the $i$-th production center to the $j$-th consumption center through the $k$-th resource distribution and storage center by the $l$-th type transmitting element; $\widetilde{\mathrm{x}}_{\mathrm{ijklr}}$ is a fuzzy set that determines the fuzzy amount of the corresponding resource.

The fuzzy objective function (2) is bounded below on the

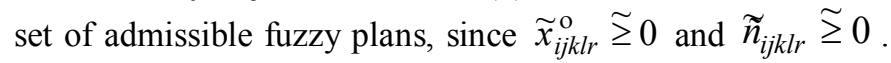
If the parameters are fuzzy defined, the constraints of the problem have the form:

$$
\begin{aligned}
& \sum_{i=1}^{I} \sum_{j=1}^{J} \sum_{k=1}^{K} \sum_{l=1}^{L} \sum_{r=1}^{R} \widetilde{x}_{i j k l r} \stackrel{\widetilde{\leq}}{\leq} \widetilde{a}_{i}, i=1,2, \ldots, I, \\
& \sum_{i=1}^{I} \sum_{j=1}^{J} \sum_{k=1}^{K} \sum_{l=1}^{L} \sum_{r=1}^{R} \widetilde{x}_{i j k l r} \cong \widetilde{b}_{j}, j=1,2, \ldots, J, \\
& \sum_{i=1}^{I} \sum_{j=1}^{J} \sum_{k=1}^{K} \sum_{l=1}^{L} \sum_{r=1}^{R} \tilde{x}_{i j k l r} \underset{\widetilde{s}}{\widetilde{d}}, k=1,2, \ldots, K, \\
& \sum_{i=1}^{I} \sum_{j=1}^{J} \sum_{k=1}^{K} \sum_{l=1}^{L} \sum_{r=1}^{R} \tilde{x}_{i j k l r} \underset{\leq}{\leq} \widetilde{q}_{l}, l=1,2, \ldots, L, \\
& \sum_{i=1}^{I} \sum_{j=1}^{J} \sum_{k=1}^{K} \sum_{l=1}^{L} \sum_{r=1}^{R} \tilde{x}_{i j k l r} \widetilde{\sim}_{r}, r=1,2, \ldots, R, \\
& (i, j, k, l, r) \in E=I \times J \times K \times L \times R,
\end{aligned}
$$

here, $\widetilde{\leq}$ - fuzzy inequality; $\cong$ - fuzzy equality; $\widetilde{a}_{i}, i \in I$ is a fuzzy set defined on the set of real numbers $\Theta_{a}$ and defining a fuzzy amount of the resource available in the $i$-th production center; $\tilde{b}_{j}, j \in J$ is a fuzzy set defined on the set of real numbers $\Theta_{b}$ and defining the fuzzy amount of resource required by the $j$-th consumption center; $\widetilde{v}_{r}$, $r \in R$ is a fuzzy set defined on the set of real numbers $\Theta_{v}$ and defining an unclear number of $r$-th resource; $\widetilde{q}_{l}, \quad l \in L$ is a fuzzy set defined on a set of real numbers $\Theta_{q}$ and defining a fuzzy amount of a resource that can be transported by a $l$-type transmitting element (load-carrying capacity); $\widetilde{d}_{k}$, $k \in K$ is a fuzzy set defined on the set of real numbers $\Theta_{d}$ and defining the fuzzy capacity of the corresponding centers.

Fuzzy intervals $\widetilde{c}_{i j k l r}, \tilde{a}_{i}, \tilde{b}_{j}, \tilde{v}_{r}, \tilde{q}_{l}$, and $\tilde{d}_{k}$ will be called fuzzy parameters of the task, and the set of fuzzy intervals $\left\{\tilde{x}_{i j k l r}\right\}$ will be called a set of fuzzy variables of the task, which completely define the five-index problem and are called the fuzzy task plan. Each fuzzy plan corresponds to a certain value of the fuzzy objective function (2). If there is a fuzzy plan that ensures the minimum fuzzy value of the objective function on the set of all admissible fuzzy plans, then this fuzzy plan is the optimal fuzzy plan of the problem. 


\section{DEVELOPMENT OF THE METHOD FOR SOLVING THE PROBLEM}

The basis of the proposed method for solving the multiindex distribution task under uncertainty source data association method is null transformation [11] to solve the three-index transportation problems and fuzzy-interval approach when setting the parameters of the problem [9].

The solution of this problem is determined by the following stages:

Stage 1. Define the minimal elements $\tilde{\alpha}_{i}, \widetilde{\beta}_{j}, \tilde{\gamma}_{k}, \tilde{\mu}_{l}, \widetilde{\varphi}_{r}$ in each section, given by the formulas:

$$
\begin{aligned}
& \tilde{\alpha}_{i}=\min _{j k l r}\left\{\tilde{c}_{i j k l r}\right\}, i \in\{1,2, \ldots, I\}, \\
& \tilde{\beta}_{j}=\min _{i k l r}\left\{\tilde{c}_{i j k l r}-\tilde{\alpha}_{i}\right\}, j \in\{1,2, \ldots, J\}, \\
& \tilde{\gamma}_{k}=\min _{i j l r}\left\{\tilde{c}_{i j k l r}-\tilde{\alpha}_{i}-\widetilde{\beta}_{j}\right\}, k \in\{1,2, \ldots, K\}, \\
& \tilde{\mu}_{l}=\min _{i j k r}\left\{\tilde{c}_{i j k l r}-\tilde{\alpha}_{i}-\widetilde{\beta}_{j}-\tilde{\gamma}_{k}\right\}, l \in\{1,2, \ldots, L\}, \\
& \tilde{\varphi}_{r}=\min _{i j k l}\left\{\tilde{c}_{i j k l r}-\tilde{\alpha}_{i}-\widetilde{\beta}_{j}-\tilde{\gamma}_{k}-\tilde{\mu}_{l}\right\}, r \in\{1,2, \ldots, R\},
\end{aligned}
$$

here $\widetilde{c}_{i j k l r}$ is the matrix of resource allocation tariffs; $\widetilde{\alpha}_{i}$ is the minimum element in the $i$-th section; $\widetilde{\beta}_{j}$ is the minimal element in the $j$-th section; $\tilde{\gamma}_{k}$ is the minimal element in the $k$-th section; $\tilde{\mu}_{l}$ is the minimum element in the $l$-th section; $\tilde{\varphi}_{r}$ is the minimal element in the $r$-th section.

Stage 2. The zero index elements of the matrix are defined:

$$
\widetilde{c}^{*}{ }_{i j k l r}=\widetilde{c}_{i j k l r}-\left(\widetilde{\alpha}_{i}+\widetilde{\beta}_{j}+\widetilde{\gamma}_{k}+\widetilde{\mu}_{l}+\widetilde{\varphi}_{r}\right) .
$$

As a result of the transformations, we obtain a matrix in which each section contains at least one zero index element $\widetilde{c}_{i j k l r}^{*}=0$ :

$$
\begin{aligned}
& \min _{j k l r}\left\{\tilde{c}^{*} i j k l r\right\}=0, i \in\{1,2, \ldots, I\}, \\
& \min _{i k l r}\left\{\tilde{c}^{*}{ }_{i j k l r}\right\}=0, j \in\{1,2, \ldots, J\}, \\
& \min _{i j l r}\left\{\tilde{c}^{*}{ }_{i j k l r}\right\}=0, k \in\{1,2, \ldots, K\}, \\
& \min _{i j k r}\left\{\tilde{c}^{*} i j k l r\right\}=0, l \in\{1,2, \ldots, L\}, \\
& \min _{i j k l}\left\{\mathcal{c}^{*} i j k l r\right\}=0, r \in\{1,2, \ldots, R\}, \\
& \tilde{c}^{*}{ }_{i j k l r} \geq 0 .
\end{aligned}
$$

Stage 3. Define the leading index elements among the zero index elements of the matrix $\widetilde{c}_{i j k l r}^{*}$. To determine the leading index element for each zero index element of set $Z^{0}=[(i j k l r)]$, it is determined $\tilde{x}_{i j k l r}$, which is equal to the minimum value of the restrictions for the corresponding indices ( $i j k l r)$. Among the minimum values of constraints corresponding to the zero index elements $\tilde{x}_{i j k l r}$, the maximum value is selected, and the corresponding value $\tilde{x}_{i j k l r}^{\prime}$ is defined as the leading index element. The set of leading index elements corresponds to the values of the matrix, which subsequently determines the optimal value of the resource allocation cost function.

Stage 4. As a result of the implementation of the transformations (3) - (5), a set of zero elements $Z^{0}=[($ ijklr $)]$ will be obtained for which the value $\widetilde{c}^{*} i j k l r=0$. As a result of reduction, the resulting matrix contains $t \geq \max \{I, J, K, L, R\}$ zero index elements. This property allows selecting the leading index element at each step in such a way that the value $\tilde{x}_{i j k l r}$ chosen at this step will be the largest.

For each index element from the set $Z^{0}=[(i j k l r)]$, a value $\tilde{x}_{i j k l r}$ is defined that is equal to the minimum value of the restrictions specified in step 1 , according to the corresponding indices $(i j k l r)$.

Stage 5. If the value of the variable $\tilde{x}_{i j k l r}$ in the crosssection $\tilde{x}_{i j k l}^{r}$ is chosen, it is necessary to exclude the crosssection $\widetilde{c}^{*}{ }_{i j k l}^{r}$ before the next step of the computational procedure from the matrix $\widetilde{c}^{*}{ }_{i j k l r}$, and to repeat the procedure for reducing the matrix.

Stage 6. The objective function is formed by summing the products of the leading index element and its corresponding value $\widetilde{c}_{i j k l r}$.

Steps 1-6 are repeated until all sections are crossed out at some index, indicating that the resource corresponding to the index is exhausted.

Sequential application of this procedure allows achieving a minimum increment value of the objective function for distributed resource unit in each calculation iteration.

The results of experiments to evaluate the effectiveness of the developed method are described in detail in [10]. The following results of the experiments can be noted:

- to solve the problem of resource allocation method of potentials must be 4 times more iterations than in the solution of this problem by the proposed method;

- time solving the problem of the proposed method is less than the average for 2000 seconds at task $10^{6}$ dimension;

- if the dimension of the task is increased, the discrepancy determined by the ratio of the values of the objective function by the proposed method and the method of potentials is reduced. With the dimension of problem $10^{6}$, the discrepancy is $0.5 \%$.

Using the potential of the method for solving large-scale problems require time-consuming, which is unacceptable for operational planning tasks. The developed method allows to avoid the problem of degeneracy in solving problems of 
distributive type, provides a solution to a problem of large dimension for a permissible time, taking into account the uncertainty of the initial parameters of the problem.

\section{DEVELOPMENT OF INFORMATION SUPPORT}

Practical application of methods for solving distribution tasks occurs within the framework of the operation of information and control systems of enterprises or organizations. The organizational structure of information management systems is directly related to the structure of enterprises or organizations, and also depends on the number and types of tasks to be accomplished. Rational management and optimization of the multifaceted activity of the distribution system cannot be achieved without the use of modern information technologies. The analysis showed that at present there are several directions of software implementations on the market (MRP (Material Requirements Planning), ERP (Enterprise Resource Planning), SCM (Supply Chain Management)), that solve individual problems of the resource distribution system of various types [12].

Existing software products are similar in many respects and have separate individual features. All of them have the following disadvantages: lack of opportunity to work with informal processes, lack of a function to assess the quality of planned decisions, lack of solutions to strategic planning tasks. The main reason, which limits the possibility of using known solutions in the field of real transport-distribution tasks, is the uncertainty of the initial data.

The software application in the programming environment $1 \mathrm{C}$ v8.3 was developed on the basis of the proposed method for solving the problem of resource allocation under uncertainty conditions. At the initial stage of working with the software application, the database is populated with input parameters. For this, the standard service of the $1 \mathrm{C}$ "Counterparty" package was modified, which allowed entering initial parameters in an unclear form (1). Based on the set parameters, automatic calculation of the preliminary cost of resource allocation is performed, which, if necessary, can be corrected by the expert. An example of setting parameters of the cost of transportation based on expert data is presented in Fig. 1.

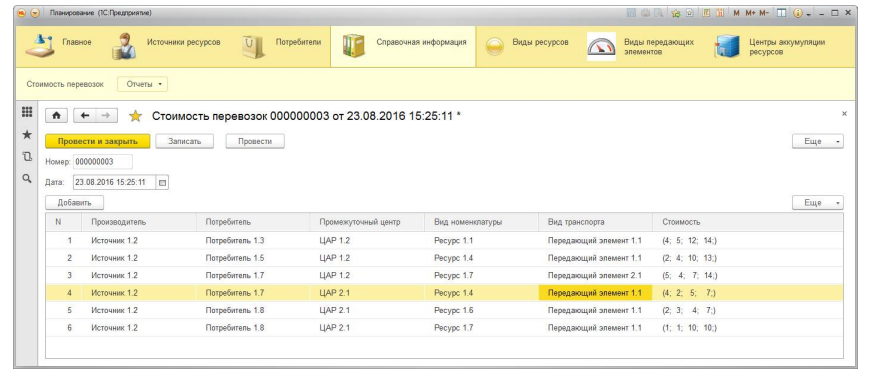

Fig. 1. Database of the cost of transportation of resources (tariffs)

The expert knowledge base is intended for long-term data storage, and also due to the available data transfer capability, the user has the possibility of increasing them for further use and analysis.
After entering the initial parameters, the optimal allocation of resources is calculated. The results can be presented to the user in the form of a report on the parameter to be set. An example is shown in Fig. 2:

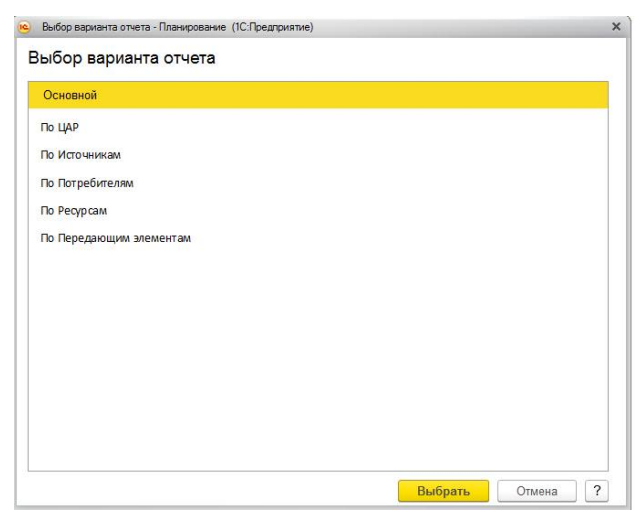

Fig. 2. Example of report selection by parameter

As a result, the user is provided with recommendations for optimizing resource allocation. An example of recommendations is shown in Fig. 3:

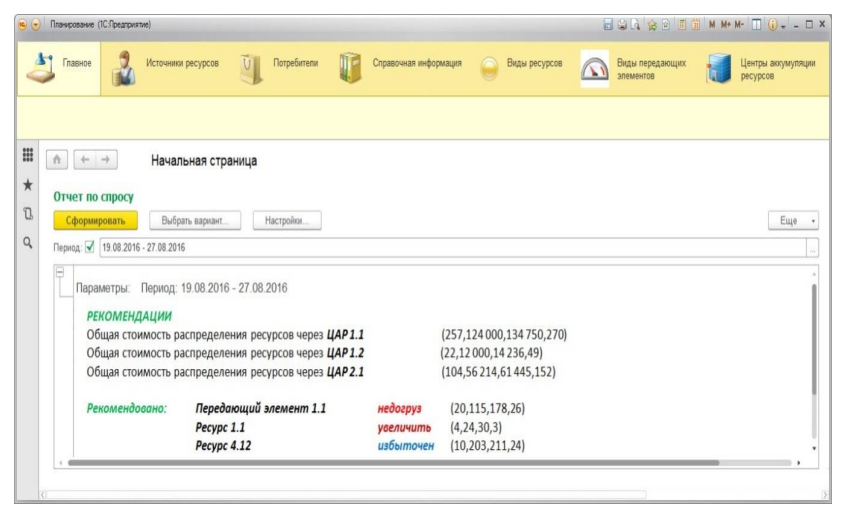

Fig. 3. Example of recommendations

Thus, the result of the work of this software application is the recommendations that provide an optimal solution to the problem of resource allocation, in order to meet consumer demand, allowing making decisions on operational planning and management of material, information and other flows.

The solution of the problem by the proposed method for fuzzy interval control of the parameters completely covers the results of the solutions in a clear formulation, which agrees with the basic principle of fuzzy-interval mathematics - the coverage of all possible solutions [14].

\section{CONCLUSION}

The task of resource allocation lies in the optimal functioning of the system, allowing efficient decision-making on the organization and operational planning of production and distribution processes.

The proposed method for finding the solution of multiindex distribution problems can be used as a replacement for the method of potentials. The application of the developed 
method does not require the construction of a problem support plan, which allows you to avoid the problem of degeneracy in problems of this class and avoid cycling when finding the best plan for moving resources.

Algorithms and methods that are applicable to solving distributive problems have many applications. The proposed fuzzy-interval method for describing the parameters of the problem corresponds to the intuitive views of experts on the predicted parameters and is a convenient tool for formalizing the initial data of the distribution problem.

The results allow judging the high efficiency of the developed method for solving multi-index problem of resource allocation under uncertainty.

The optimal solution obtained with the help of the developed method is very informative, which makes it possible to talk about the prospects of using fuzzy interval analysis in the modeling of distribution tasks.

\section{REFERENCES}

[1] V. Uzun, V. Saraikin, E. Gataulina, N. Shagayda, R. Yanbykh, S. Mary, S. GomezyPaloma: Prospects of the farming sector and rural development in view of food security, Luxembourg. Publications Office of the European Union, 2014, 138p.

[2] Supply Chain Management. Edited by Dr. Pengzhong Li, Publisher InTech, 2011, 590p.

[3] Leung J.Y.-T. (ed.) Handbook of Scheduling. Algorithms, Models, and Performance Analysis. Chapman\&Hall/CRC, 2004, 1157p.

[4] O.V.Kosenko. Improving the efficiency of methods for solving multiobjectives resource allocation. Izvestiya SFedU. Engineering sciences, 2016, V. 4(177), pp.73-81.

[5] Z. I. Botev The normal law under linear restrictions: simulation and estimation via minimax tilting. Journal of the Royal Statistical Society. Series B (Statistical Methodology), 2017, V.1(79), pp. 125-148.

[6] K.Ashraf. A Logistical Approach to Managing the Resources of Multi Nomenclature Spare Parts of a Corporate Car Service in Conditions of Risk and Uncertainty of Demand. Science Journal of Business and Management, 2017, V. 5(4), pp.169-174.

[7] V.V.Dombrovsky. Dynamic network model of inventory management with interval uncertainty of demand. Computing Technologies, 2001, V. 4, pp.271-174

[8] O.Kosenko, E.Sinyavskaya, E.Shestova, E.Kosenko, S.Antipin. Method of rational placement of intermediate centers with setting parameters in the form of the fuzzy intervals. In the Proceedings of the 19th International Conference on Soft Computing and Measurements (SCM 2016), 2016. pp. $186-189$.

[9] D. Dubois and H. Prade. Possibility Theory: An Approach to Computerized Processing of Uncertainty. New York: Plenum Press. 1988, 263p.

[10] O.Kosenko, E.Sinyavskaya, E.Shestova, E.Kosenko, O.Chemes. Method for solution of the multi-index transportation problems with fuzzy parameters. In the Proceedings of the 19th International Conference on Soft Computing and Measurements (SCM 2016), 2016, pp. $179-182$.

[11] L.Raskin, I.Kirichenko. Multi-index problems of linear programming. Moscow: Radio and Communication. 1982. 240 p.

[12] O.Leary, L.Daniel. Enterprise resource planning systems. Cambridge University Press, 2000, 232 p.

[13] 1C: Enterprise 8. Moscow: Firm "1C". 2012, 312 p.

[14] O.Kosenko, E.Shestova, E.Sinyavskaya, E.Kosenko, A.Nomerchuk, A.Bozhenyuk. Development of information support for the rational placement of intermediate distribution centers of fuel and energy resources under conditions of partial uncertainty. In the Proceedings of
2017 20th IEEE International Conference on Soft Computing and Measurements (SCM 2017), 2017,pp. $136-141$. 